\title{
Modeled Microgravity Affects Fibroblast Functions Related to Wound Healing
}

\author{
Francesca Cialdai $^{1}$ - Leonardo Vignali ${ }^{1} \cdot$ Lucia Morbidelli $^{2} \cdot$ Alessandra Colciago $^{3}$. \\ Fabio Celotti ${ }^{3}$ - Alice Santi ${ }^{4}$ - Anna Caselli ${ }^{4}$. Paolo Cirri ${ }^{4}$ • Monica Monici ${ }^{1}$
}

Received: 25 January 2016 / Accepted: 18 December 2016 / Published online: 18 January 2017

(c) Springer Science+Business Media Dordrecht 2017

\begin{abstract}
Wound healing is crucial for the survival of an organism. Therefore, in the perspective of space exploration missions, it is important to understand if and how microgravity conditions affect the behavior of the cell populations involved in wound healing and the evolution of the process. Since fibroblasts are the major players in tissue repair, this study was focused on the behavior of fibroblasts in microgravity conditions, modeled by a RCCS. Cell cytoskeleton was studied by immunofluorescence microscopy, the ability to migrate was assessed by microchemotaxis and scratch assay, and the expression of markers of fibroblast activation,
\end{abstract}

This article belongs to the Topical Collection: Advances in gravityrelated phenomena in biological, chemical and physical systems Guest Editors: Valentina Shevtsova, Ruth Hemmersbach

Electronic supplementary material The online version of this article (doi:10.1007/s12217-016-9532-7) contains supplementary material, which is available to authorized users.

Monica Monici

monica.monici@unifi.it

1 ASAcampus Joint Laboratory, ASA Research Division, Department of Experimental and Clinical Biomedical Sciences "Mario Serio", University of Florence, Viale G. Pieraccini 6, 50139 Florence, Italy

2 Department of Life Sciences, University of Siena, Via A. Moro 2, 53100 Siena, Italy

3 Department of Pharmacological and Biomolecular Sciences, University of Milano, Via Balzaretti 9, 20133, Milano, Italy

4 Section of Biochemistry, Department of Experimental and Clinical Biomedical Sciences "Mario Serio", University of Florence, Viale Morgagni 50, 50134 Florence, Italy angiogenesis, and inflammation was assessed by western blot. Results revealed that after cell exposure to modeled microgravity conditions, a thorough rearrangement of microtubules occurred and $\alpha$-SMA bundles were replaced by a tight network of faulty and disorganized filaments. Exposure to modeled microgravity induced a decrease in $\alpha$-SMA and E-CAD expressions. Also, the expression of the pro-angiogenic protein VEGF decreased, while that of the inflammatory signal COX-2 increased. Fibroblast ability to adhere, migrate, and respond to chemoattractants (PRP), closely related to cytoskeleton integrity and membrane junctions, was significantly impaired. Nevertheless, PRP was able to partially restore fibroblast migration.

Keywords Modeled microgravity $\cdot$ In vitro wound-healing model · Fibroblasts $\cdot$ Cell migration · Cytoskeleton ·

Inflammation markers $\cdot$ Rotary cell culture system
Abbreviation List:
ADU Arbitrary densitometric units
AJs Adherens junctions
BCA Bicinchoninic acid
CFDA-SE Carboxyfluorescein diacetate succinimidyl ester
COX-2 Cyclooxygenase-2
DMEM Dulbecco's modified Eagle's medium
E-CAD Epithelial cadherin
ECM Extracellular matrix
EGF Epidermal growth factor
EPC Endothelial precursor cells
FBS Fetal bovine serum
FGF Fibroblast growth factor
FITC Fluorescein isothiocyanate
HGF Hepatocyte growth factor
HRP Horseradish peroxidase 
IL-1 Interleukin 1

IL-10 Interleukin 10

IL-6 Interleukin 6

IL-8 Interleukin 8

INF- $\gamma$ Interferon gamma

MMPs Matrix metalloproteinases

PBS Phosphate-buffered saline

PDGF Platelet-derived growth factor

PRP Platelet-rich plasma

PVDF Polyvinylidene difluoride

RCCS Rotary Cell Culture System

RPM Random Positioning Machine

SDF-1 Stromal-derived factor-1

TGF- $\beta$ Transforming growth factor-beta

TNF- $\alpha$ Tumor necrosis factor alpha

TNF- $\beta$ Tumor necrosis factor beta

VEGF Vascular endothelial growth factor

$\alpha$-SMA Alpha smooth muscle actin

\section{Introduction}

In recent reports on the "state of the art" in medical and surgical care during space missions, wound healing has been regarded as a critical point, because it has been poorly studied and needs to be further investigated (Drudi et al. 2012). Wound healing is a crucial process for survival: it starts immediately after injury with hemostasis and aims at re-establishing the epithelial barrier. After hemostasis, it proceeds through three sequential steps, partly overlapped:

i) Inflammation, in which bacteria and cell debris are removed from the wound and soluble factors are released that induce cell migration and also stimulate cell division in the next phase;

ii) Proliferation, that is characterized by angiogenesis, collagen deposition, granulation tissue formation, epithelialization and wound contraction;

iii) Remodeling, in which collagen is remodeled and realigned along tension lines while apoptosis and phagocytosis of apoptotic bodies lead to the removal of unnecessary cells.

The whole process is fragile and susceptible to interruption, failure or alteration, leading to the formation of non-healing chronic wounds or fibrotic scars and increasing the risk of infections, ultimately causing patient morbidity.

Stromal activation has a fundamental role in wound healing: during the inflammatory response generated by traumatic tissue injury, a cytokine-mediated crosstalk starts between immune and stromal cells, which leads to fibroblast activation and many other downstream effects, such as epithelial cell proliferation, neo-angiogenesis, etc.
Fibroblasts represent the major cellular component of the stroma and, together with keratinocytes and endothelial cells, are the protagonists of tissue repair. In response to trauma and inflammation, resident fibroblasts or circulating fibrocytes transdifferentiate into myofibroblasts, their activated counterpart. In particular, during the inflammatory phase, platelets and endothelial and immune cells are present in the wound microenvironment, where they release pro-inflammatory mediators. Among them, there are growth factors, including platelet-derived growth factor (PDGF), tumor necrosis factor (TNF)- $\alpha$ and TNF- $\beta$, hepatocyte growth factor (HGF), transforming growth factor- $\beta$ (TGF- $\beta$ ), epidermal growth factor (EGF), and fibroblast growth factor (FGF), cytokines, such as interleukin (IL)1, IL-6, IL-8, and IL-10, and interferon gamma (INF- $\gamma$ ) (Wong and Crawford 2013; Peplow and Chatterjee 2013).

Many growth factors are implicated in fibroblast proliferation and migration (PDGF and FGF) and in their differentiation into myofibroblasts (TGF- $\beta$ and IL-6). Myofibroblasts, in turn, engage a crosstalk with other cell types in the microenvironment by secreting various cytokines, whose action leads to the recruitment of endothelial precursor cells (EPC) via stromal-derived factor-1 (SDF-1) (Werner and Grose 2003; Bermudez et al. 2011) and neoangiogenesis stimulation via vascular endothelial growth factor (VEGF) upregulation (Wilgus and DiPietro 2012).

Fibroblasts are the main cell type involved in the regulation of extracellular matrix (ECM) protein production and ECM remodeling through matrix metalloproteinases (MMPs) secretion. Following injury, myofibroblasts regulate connective tissue remodeling by combining their ability of ECM biosynthesis with cytoskeletal characteristics of contractile smooth muscle cells. ECM stiffness along with the action of TGF- $\beta 1$ are the most important factors that induce myofibroblast differentiation and persistence. In turn, the contractile activity of myofibroblasts increases tissue stiffness, generating the conditions that make myofibroblasts even more contractile, thus causing a feed-forward loop that could become detrimental (Grinnell and Petroll 2010) and lead to fibrotic scars. When myofibroblast activity becomes excessive, beneficial tissue repair turns into the pathological condition called organ fibrosis.

It has become clear that skin is a mechanosensitive tissue (Evans et al. 2013), and quality and speed of healing are regulated by many types of mechanical forces, such as compression, tension, shear stress, osmotic pressure, and gravity, which intrinsically and extrinsically stimulate fibroblasts, myofibroblasts, and endothelial and epithelial cells in granulation tissues (Agha et al. 2011; Hinz et al. 2001a; Couchman et al. 1990).

Some studies reported that exposure to microgravity may induce skin atrophy (Neutelings et al. 2015), delay and 
impair wound healing (Davidson et al. 1999; Delp 2008; Radek et al. 2008), and alter processes involved in it, such as inflammation, apoptosis, cell migration, collagen formation, and ECM deposition (Infanger et al. 2006; Pietsch et al. 2011; Morbidelli et al. 2005; Monici et al. 2006, 2011; Chung and Erickson 1997).

Other studies revealed that fibroblast behavior is strongly affected by loading conditions. Seitzer et al. (1995) found that collagen biosynthesis by human dermal fibroblasts increased and decreased in unloading and loading conditions, respectively. In WI-38 fibroblasts, space flight induced changes in gene expression, mostly associated with cellular stress signaling, directing the cells to either apoptotic death or premature senescence (Liu and Wang 2008). Following exposure to microgravity, modeled by a Random Positioning Machine (RPM), fetal fibroblasts showed alterations in distribution of $\alpha 5 \beta 1$ integrins and the network of actin filaments (Monici et al. 2011). Culturing fetal murine fibroblasts in a RPM induced oxidative stress-responsive genes and downregulated genes involved in cytoskeleton organization and adhesion (Beck et al. 2014).

The study reported here aimed to investigate the impact of modeled microgravity on fibroblast functions relevant to wound healing.

\section{Materials and Methods}

\section{Reagents}

Reagents used for cell culture and Cytodex 3 beads were purchased from Sigma-Aldrich (Chemical Co St Louis, MO, USA). Carboxyfluorescein diacetate succinimidyl ester (CFDA-SE) was purchased from Merck-Millipore (Darmstadt, Germany). Antibodies used in immunofluorescence analysis were anti- $\alpha$-tubulin from Upstate Biotechnology (Darmstadt, Germany), anti- $\alpha$-smooth muscle actin ( $\alpha$-SMA) from Merck-Millipore (Darmstadt, Germany), and anti-mouse IgG from Chemicon Int. (Temecula, CA, USA). Antibodies used in western blot analysis include the following: anti-cycloxygenase-2 (COX-2) from Cayman Chemical Company (Ann Arbor, MI, USA), antiVEGF from BIOSS (Bioclass, Pistoia, Italy), anti- $\beta$-actin and anti- $\beta$-tubulin from Sigma-Aldrich (St. Louis, MO, USA), anti- $\alpha$-smooth muscle actin ( $\alpha$-SMA), and anti-E cadherin (E-CAD) from Santa Cruz Biotechnology Inc. (Heidelberg, Germany). Polyvinylidene difluoride (PVDF) membrane and Immobilon Western Chemiluminescent HRP Substrate were purchased from Merck-Millipore (Darmstadt, Germany). Calcium gluconate/batroxobine used for platelet-rich plasma (PRP) preparation was purchased from Pentapharm (Basel, Switzerland).

\section{Cell Culture}

Fibroblasts (NIH-3T3) were routinely cultured in Dulbecco's modified Eagle's medium supplemented with 100 $\mu \mathrm{g} / \mathrm{ml}$ streptomycin, $100 \mathrm{U} / \mathrm{ml}$ penicillin, $2 \mathrm{mM}$ glutamine, and $10 \%$ fetal bovine serum (FBS). Cells were incubated at $37{ }^{\circ} \mathrm{C}$ in humidified atmosphere containing $95 \%$ air and $5 \%$ $\mathrm{CO}_{2}$.

\section{Modeling of Microgravity Conditions}

Microgravity conditions were modeled by a Rotary Cell Culture System (RCCS; Synthecon) equipped with 10 and $50 \mathrm{ml}$ autoclavable culture vessels. The RCCS is a horizontally rotating, bubble-free culture vessel with membrane diffusion gas exchange. Cell sedimentation in the vessel is offset by the rotating fluid, creating a constant, gentle fall of cells through the medium. For a more detailed description of the instrument, see http://synthecon.com/pages/rotary cell_culture_systems_13.asp, https://www.nasa.gov/offices/ oct/home/tech_life_synthecon.html\#.VzBul-TrfKN, http:// www.nasa.gov/vision/earth/technologies/f_spacecells.html, and Electronic Supplementary Material 1.

RCCS is one of the most frequently used ground-based simulators of microgravity. Although in the scientific community the discussion about capacities and limitations of the different microgravity simulators is still open, a recent review that evaluated the various microgravity simulators, using organism/cell extensively studied both in simulated and real microgravity, concluded that $2 \mathrm{D}$ and $3 \mathrm{D}$ clinostats are valuable tools for simulating microgravity conditions in adherent mammalian cells (Herranz et al. 2013).

In order to be used in the RCCS, adherent cells need to be cultured on microcarrier beads (Cytodex 3 ). For the experiments described here, $8 \times 10^{5}$ cells were seeded in Petri dishes (polystyrene plates, $100 \mathrm{~mm}$ diameter, $20 \mathrm{~mm}$ height) and allowed to grow until they reached $95 \%$ confluency. Cells were then incubated with beads $(5 \mathrm{mg} / \mathrm{ml}$ culture medium) and allowed to adhere to them. After $24 \mathrm{~h}$, vessels were filled with medium containing beads with cells adhering to the surface and placed on the RCCS for $72 \mathrm{~h}$. The RCCS was placed inside a dedicated incubator $\left(37^{\circ} \mathrm{C}\right.$, humidified atmosphere with $5 \% \mathrm{CO}_{2}$ ). Control vessels $(1 \times g)$ were prepared accordingly and placed inside the incubator, at the base of the RCCS. Rotation speed was set at 20 $\mathrm{rpm}$, following manufacturer suggestions and preliminary experiments.

\section{Platelet-Rich Plasma Preparation and Activation}

Platelet-rich plasma was obtained from blood of normal healthy volunteers and prepared as previously described 
(Celotti et al. 2006). Briefly, blood was collected using citrate phosphate dextrose as anti-coagulant. The whole blood was centrifuged at $180 \times \mathrm{g}$ for $20 \mathrm{~min}$ : platelets were in the upper layer, while red blood cells and white blood cells were in the lower layer. The upper layer was transferred into clean tubes and centrifuged at $580 \times \mathrm{g}$ for $15 \mathrm{~min}$ to create a precise separation of the platelet pellets from the platelet-poor plasma (PPP). A small volume of PPP was used to resuspend the platelet pellets, giving the final PRP fraction (platelet enrichment of about four to five times and $1.0-1.2 \times 10^{6}$ platelets/ $\mu$ l on average), which was activated into a sterile syringe using calcium gluconate/batroxobine (Pentapharm, Basel, Switzerland). One to three minutes after batroxobine addition, this mixture solidified as batroxobine induces fibrin polymerization into an insoluble gel; at the same time, platelets degranulated releasing growth factors and cytokines, which remained trapped in the gel. The platelet gel was centrifuged $(1400 \times g, 10 \mathrm{~min}, \mathrm{RT})$, and the liquid phase was enriched in growth factors (PRP in text and figures) and frozen at $-20{ }^{\circ} \mathrm{C}$ in aliquots till use. Under these storage conditions, the preparation remains effective for many months.

In the experiments described here, PRP diluted 1:500 was used as chemoattractant, according to previous studies in which PRP (1:500) was demonstrated to be as effective as the known chemoattractant FCS (1\%) in improving cell migration (Celotti et al. 2006).

\section{Quantification of Growth Factor Content in PRP}

The amount of different growth factors in PRP was quantified using the quantitative sandwich enzyme immunoassay technique (Quantikine Immunoassay, R\&D System, Minneapolis, USA).

\section{Immunofluorescence Microscopy}

One milliliter of suspension (medium containing beads with fibroblasts adhering to the surface) was collected from each sample, both controls $(1 \times g)$ and samples exposed for $72 \mathrm{~h}$ to modeled microgravity, and transferred to vials. After spontaneous sedimentation of the beads (with adherent cells) on the bottom of the vial, supernatant was discarded by gently aspirating with a pipette and $1 \mathrm{ml}$ of cold acetone was added for $5 \mathrm{~min}$. Once the fixative was removed, samples were washed three times with $2 \mathrm{ml}$ of PBS each, every time discarding the supernatant after beads sedimentation. After blocking unspecific binding with PBS containing $3 \%$ bovine serum albumin (BSA) for $30 \mathrm{~min}$, the cells still adherent to the beads were incubated overnight at $4{ }^{\circ} \mathrm{C}$ with the specific anti- $\alpha$-tubulin and anti- $\alpha$-SMA antibodies (diluted 1:100 in PBS with 0.5 \% BSA). After washing three times with $2 \mathrm{ml}$ of PBS as described above, samples were incubated for $1 \mathrm{~h}$ with the fluorescein isothiocyanate (FITC)-conjugated specific secondary antibody (specifically, anti-mouse IgG, diluted 1:200 in PBS with $0.5 \%$ BSA) at room temperature in the dark. Samples were washed three times with $2 \mathrm{ml}$ of PBS as above described. Finally, a small drop of suspension (containing beads with stained fibroblasts adherent to the surface) was mounted in between a glass slide and a coverslip. Negative controls were obtained by omitting the primary antibodies. Samples were evaluated by epifluorescence microscopy (Nikon, Florence, Italy), using a FITC BP filter, at $\times 100$ magnification and imaged by a HiRes IV digital CCD camera (DTA, Pisa, Italy).

\section{Western Blot}

Trypsinized cells $\left(1 \times 10^{6} /\right.$ sample $)$ were lysed with $2 \times$ SDSLaemmli sample buffer without $\beta$-mercaptoethanol and bromophenol blue, and protein concentration was evaluated by bicinchoninic acid (BCA) assay.

The proteins were separated by SDS-PAGE, transferred on PVDF membrane by Western Blot and probed with anti- $\alpha$-SMA antibody (1:1000), anti E-CAD (1:1000), antiCOX-2 (1:1000), and anti-VEGF (1:1000). Immunoblots were incubated with the respective primary antibody in phosphate-buffered saline supplemented with $5 \%$ BSA and $0.05 \%$ Tween for $1 \mathrm{~h}$ at room temperature, washed three times (10 min each) with PBS supplemented with $1 \%$ BSA and $0.05 \%$ Tween and then incubated with the respective species-specific secondary antibody conjugated with horseradish peroxidase (HRP) in PBS supplemented with $1 \%$ BSA and $0.05 \%$ Tween for $45 \mathrm{~min}$ at RT. Anti- $\beta$-actin $(1: 10000)$ and anti- $\beta$-tubulin (1:1000) were used as loading controls. The membranes were incubated with Immobilon Western Chemiluminescent HRP Substrate, and chemiluminescence was detected by Ultra-Violet Products (UVP) Ltd Chemidoc-it 500 Imaging System. Quantitative analysis of the lanes was carried out by Kodak MI software. Data were calculated as relative changes (\%) of the arbitrary densitometric units (ADU) of the target protein with respect to the proteins $\beta$-actin or $\beta$-tubulin used as loading controls.

\section{Adhesion Assay}

Cells exposed to modeled microgravity and control cells were assayed by Trypan blue exclusion test to assess cell viability; $1 \times 10^{5}$ viable cells were plated in $60-\mathrm{mm}$ dishes with complete culture medium for $30 \mathrm{~min}$ at $37{ }^{\circ} \mathrm{C}$. Then, the supernatant was removed and the plates were washed with PBS. The attached cells were detached by trypsinization and counted with Burker's chamber. 


\section{Migration from Beads to Plate Surface}

Fibroblasts attached to the beads ( $5 \mathrm{ml}$ of suspension) were seeded in 60-mm plates for $6 \mathrm{~h}$. Plates were then washed twice with PBS to remove beads and non-adherent cells. The cells migrated to the plate surface were detached with trypsin, counted, and normalized in comparison with the total cell number present on the beads.

\section{Microchemotaxis Assay}

The microchemotaxis assay was performed using a 48-well Boyden's chamber according to manufacturer's instructions (Neuroprobe, Cabin John, MD, USA). Fibroblasts were cultured in starvation medium for $24 \mathrm{~h}$. Then, cells were counted, loaded into the open-bottom wells of the upper compartment of the transwell filter, and allowed to migrate through a polyvinylpyrrolidone-free polycarbonate porous membrane $(8 \mu \mathrm{m}$ pore size). For the experiments with PRP (1:500) used as chemoattractant, the porous membrane was pre-coated with gelatine $\left(0.2 \mathrm{mg} / \mathrm{ml}\right.$ in PBS, 5 days at $\left.4{ }^{\circ} \mathrm{C}\right)$. After migration (overnight, $37^{\circ} \mathrm{C}$ ), cells adherent to the underside of the membrane were fixed by methanol and stained according to the Diff-Quik kit (Biomap, Milan, Italy). For quantitative analysis, cells were photographed using an optical microscope with a Canon camera. The number of migrating cells was evaluated by counting the cells that migrated to the lower surfaces of the filter.

\section{Scratch Assay}

Scratch assay is an in vitro model of wound healing used to study cell activation and migration in response to injury. After exposure to modeled $\mu g$, a 5-ml volume was taken from exposed samples and controls and transferred in 6well culture dishes for $48 \mathrm{~h}$. When confluent, cells were synchronized by serum depletion for $24 \mathrm{~h}$. Then, the culture medium was removed and a scratch was made on the monolayers with a plastic tip. Wounded monolayers were washed three times with $\mathrm{Ca}^{2+}$ and $\mathrm{Mg}^{2+}$ containing PBS (pH 7.4) to remove dead cells. Cell migration occurred in $3 \mathrm{ml}$ of medium with $10 \%$ FBS. When required, PRP 1:500 was added to the medium in controls $(1 \times g)$ and microgravity-exposed samples. Scratches were observed and photographed immediately after scratching and then every $12 \mathrm{~h}$, up to $48 \mathrm{~h}$, using an inverted microscope $(\times 10)$.

\section{Statistics}

Three different experiments were carried out in triplicate. Data are reported as means \pm SD.
Statistical significance was determined using two-sided Student's $t$ test. A $p$ value lower than 0.05 was considered statistically significant.

For immunofluorescence analysis, at least 30 cells per slide were scored in ten random fields/slide.

\section{Results}

\section{Rearrangement of the Cytoskeleton in Fibroblasts Exposed to Modeled $\mu \mathrm{g}$}

Cytoskeletal components and their distribution were studied by immunofluorescence microscopy. The exposure to modeled $\mu g$ induced changes in the arrangement of microtubules and $\alpha$-SMA filaments. Control cells specifically stained for $\alpha$-tubulin revealed the expected organization pattern: microtubules radially distributed from the microtubule organizing centre, near the nucleus, towards the periphery of the cell (Fig. 1a). Instead, in cells exposed to modeled $\mu g$, microtubules lost the radial distribution, they appeared convoluted and formed a dense network (Fig. 1b). In control cells ( $1 \times g)$, $\alpha$-SMA staining clearly revealed bundles running parallel to each other (Fig. 1c). After culturing in modeled $\mu g, \alpha$-SMA bundles were replaced by a tangle of faulty and disorganized filaments (Fig. 1d).

\section{Protein Expression in Fibroblasts Exposed to Modeled $\mu \mathrm{g}$}

After being exposed to conditions of modeled $\mu \mathrm{g}$ for 72 h, NIH-3T3 fibroblasts were lysed and proteins were subjected to gel electrophoresis. Controls at " $1 \times g$ " were treated in the same way, except for exposure to modeled $\mu g$. The expression of markers of fibroblast activation, angiogenesis, and inflammation was assessed by western blot. The results revealed that $\alpha$-SMA protein, which is involved in cytoskeleton organization and is considered an activation marker in fibroblasts, significantly decreased $(38 \pm 12 \%)$ in NIH-3T3 exposed to modeled $\mu g$, compared with $1 \times g$ controls (Fig. 2a). Also E-CAD, which is involved in cell adhesion and fibroblast activation, showed a decrease (15 $\pm 6 \%$ ) after exposure to modeled $\mu g$ (Fig. $2 \mathrm{~b}$ ). This result, although not statistically significant $(p>0.05)$, showed a trend consistent with the other results. Moreover, modeled $\mu g$ induced a decrease $(31 \pm 19 \%)$ in the expression of the pro-angiogenic protein VEGF (Fig. 3a), while the expression of the inflammatory signal COX-2 was increased by $87 \pm 22 \%$ (Fig. 3b). HIF-1 $\alpha$, another marker linked to angiogenesis induction in conditions of hypoxic stress, was not expressed in this cell line in both $1 \times g$ - and modeled microgravity conditions (data not shown). 
Fig. 1 Effect of modeled $\mu \mathrm{g}$ on cytoskeleton componentsimmunofluorescence microscopy: samples were stained with monoclonal anti- $\alpha$ tubulin (a, b) anti- $\alpha$-SMA (c, d), and FITC-conjugated secondary antibody; magnification, $\times 100$. a Controls $(1 \times g)$ showed radially distributed microtubules, while b in fibroblasts exposed to modeled $\mu g$, microtubules were convoluted and tangled; c controls $(1 \times g)$ showed parallel bundles of $\alpha$-SMA, while $\mathbf{d}$ in fibroblasts exposed to modeled $\mu g$, the bundles were replaced by a tangle of faulty and disorganized filaments. Bar, 10 $\mu \mathrm{m}$

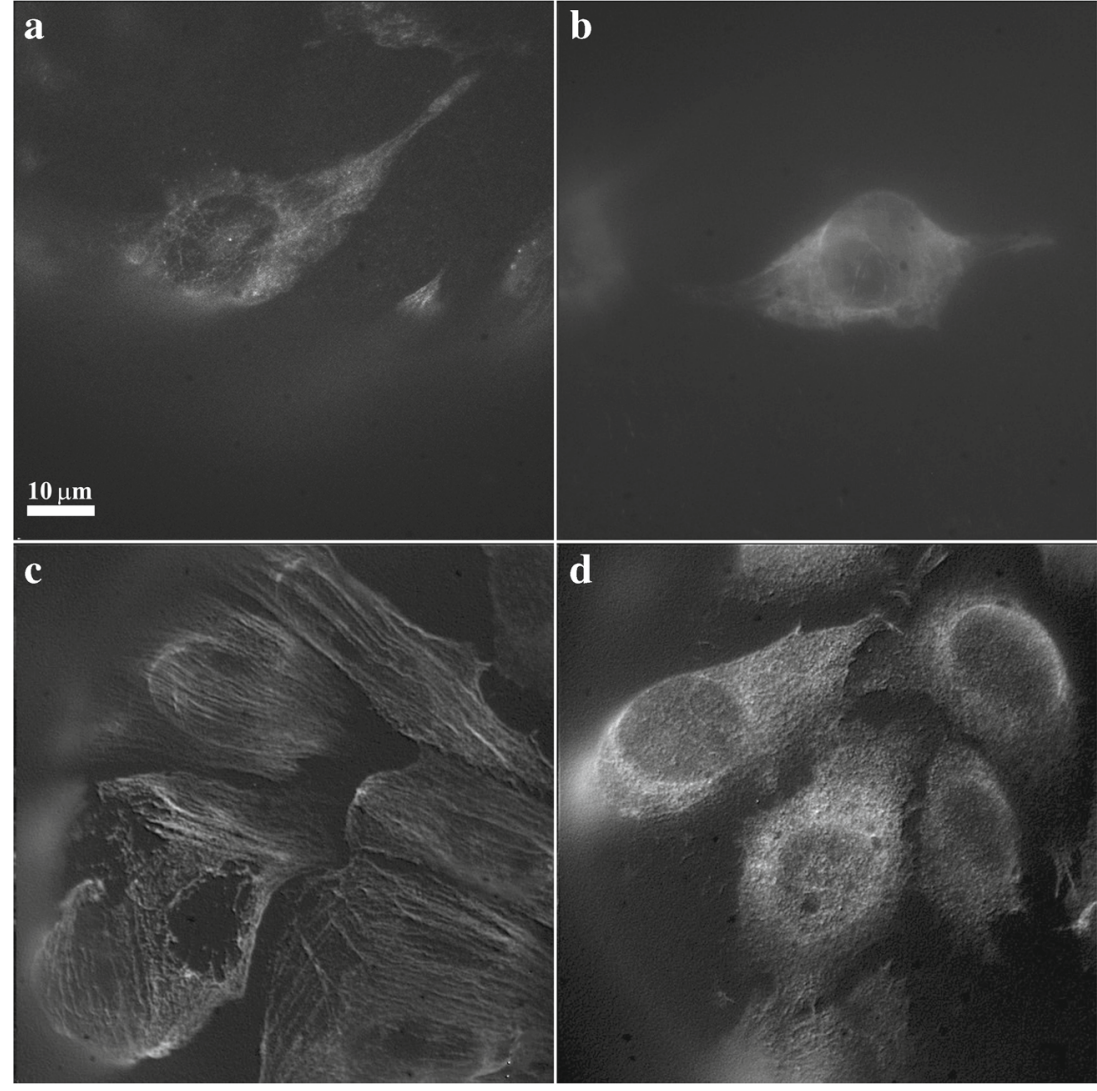

\section{Effect of Modeled $\mu \mathrm{g}$ on Cell Adhesion and Migration}

The exposure to modeled $\mu \mathrm{g}$ deeply affected both cell adhesion and migration. In comparison with $1 \times g$ controls, fibroblasts kept in modeled $\mu \mathrm{g}$ conditions for $72 \mathrm{~h}$ showed a decrease $(27 \pm 6 \%)$ in their adhesion ability (Fig. 4). In addition, modeled $\mu \mathrm{g}$ inhibited cell migration.

When assessing the ability of cells attached on beads to migrate to the plate surface, it was found that in samples kept in modeled $\mu \mathrm{g}$ the number of migrating cells was $59 \pm 10 \%$ lower than in the $1 \times g$ controls (Fig. 5a). The microchemotaxis assay confirmed this result: yet fibroblasts exposed to modeled $\mu \mathrm{g}$ for $72 \mathrm{~h}$ showed a reduced ( 15 $\pm 10 \%$ ) ability to migrate in comparison with $1 \times g$ controls (Fig. 5b), although in this case the difference was not statistically significant $(p>0.05)$.

An in vitro scratch assay was applied to study the effect of exposure to modeled $\mu \mathrm{g}$ on cell migration in response to injury. The results demonstrated that exposure to modeled $\mu \mathrm{g}$ affected the fibroblast's ability to migrate and close the scratch. After 48 h, $58 \%$ of the scratched area was closed in the control samples $(1 \times g)$ (Fig. 6a, b and e). In cell cultures previously exposed to modeled $\mu \mathrm{g}$, the closing proceeded more slowly: after $48 \mathrm{~h}$, the scratched area was closed by $24 \%$ only (Fig. 6c-e), with a significant difference (34 $\pm 7 \%)$ compared with $1 \times \mathrm{g}$ controls.

\section{Effect of PRP on Fibroblasts Exposed to Modeled $\mu \mathrm{g}$}

Many growth factors, cytokines, and other bioactive compounds are released by platelets upon activation when wound healing is initiated in either soft or in bone tissue, and many of them are present in PRP preparations (for the mean concentration of the growth factors present in the PRP, see Colciago et al. 2009). The most abundant are PDGF, present as homodimers PDGF-AA and PDGF-BB, and as the heterodimer PDGF-AB, TGF- $\beta$, mainly $\beta 1$, and the insulin-like growth factor (IGF)-1. NIH-3T3 cells exposed to modeled $\mu \mathrm{g}$ for $72 \mathrm{~h}$ were less responsive to PRP (1:500) chemoattraction $(27 \pm 11 \%)$ than $1 \times g$ controls (Fig. 7). However, the addition of PRP to the culture medium immediately after the exposure to unloading conditions was able to restore, at least in part, the chemokinetic properties compromised by modeled microgravity exposure, as evaluated by scratch assay (Fig. 8).

\section{Discussion}

Fibroblasts play a pivotal role in wound healing, and therefore, studies on their activation, migration and transdifferentiation into myofibroblasts have strongly contributed to a better understanding of the molecular and cellular 


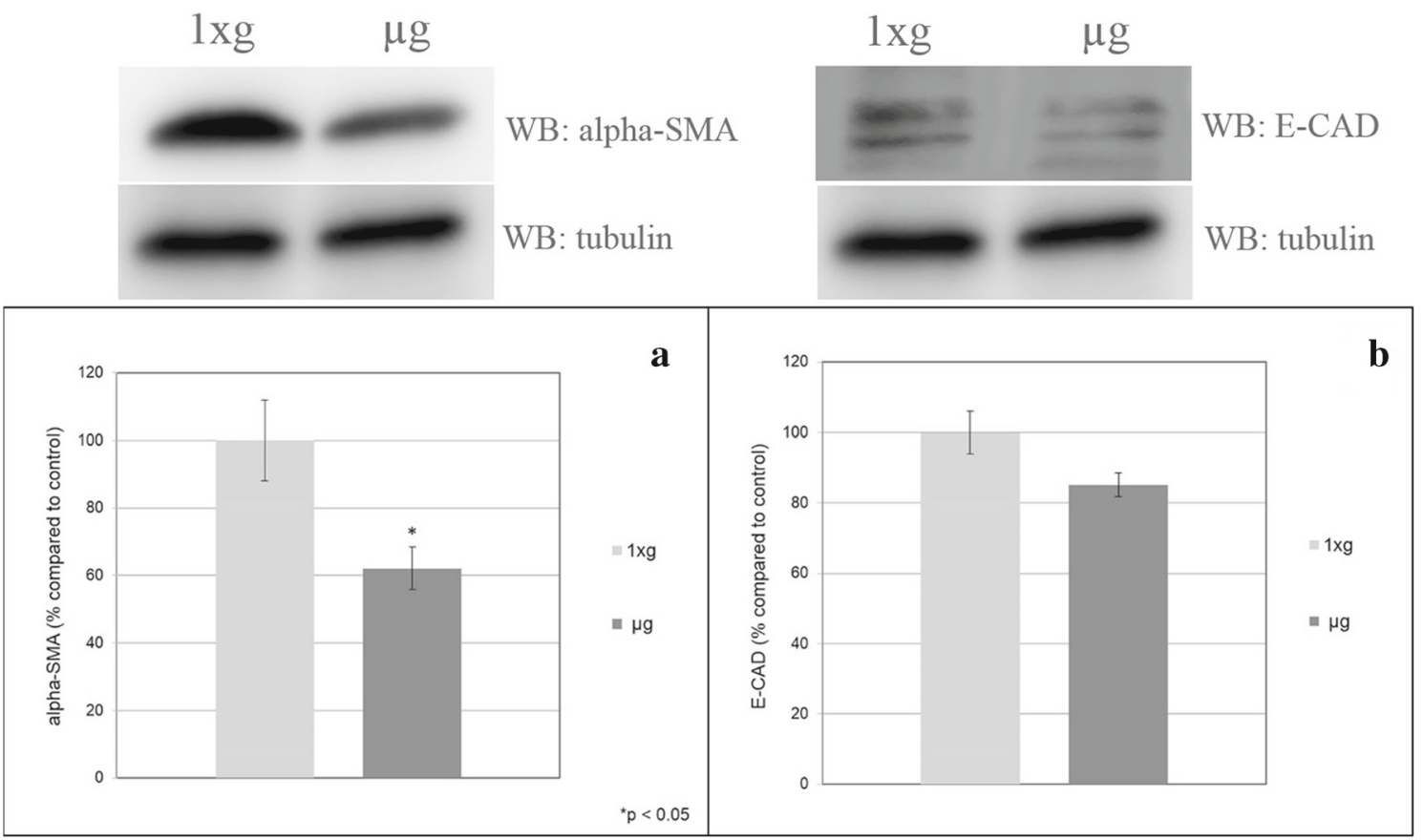

Fig. 2 Protein expression assessed by western blot. a The expression of $\alpha$-SMA, a cytoskeletal protein considered an activation marker in fibroblasts, decreased by $38 \%(p<0.05)$ in fibroblasts exposed to modeled $\mu g$, compared with $1 \times g$ controls; b also E-CAD, a

mechanisms underlying tissue repair and remodeling on Earth $(1 \times g)$.

Several reports have raised concerns about woundhealing impairment in $\mu g$ (Davidson et al. 1999; Delp 2008; Radek et al. 2008). Fibroblasts are known to be mechanosensitive cells (Gabbiani 2003), and changes in gene expression profile have been found in fibroblasts exposed to unloading conditions (Liu and Wang 2008; Beck et al. 2014), thus suggesting the hypothesis that $\mu g$-induced protein involved in cell adhesion, showed $15 \%$ decrease after exposure to modeled $\mu \mathrm{g}$. E-CAD decrease, although not statistically significant $(p>0.05)$, showed a trend consistent with the other results obtained in the study

alterations in fibroblast behavior can be a major cause of wound-healing failure in weightlessness. In order to verify this hypothesis, morphological features and functional aspects relevant to tissue repair were studied in fibroblasts exposed to $\mu g$ modeled by a RCCS.

After the exposure, an impressive rearrangement of the cytoskeleton was observed: radially distributed microtubules became convoluted and tangled, while parallel bundles of $\alpha$-SMA were replaced by a tangle of faulty and
Fig. 3 Protein expression assessed by western blot. a In fibroblasts exposed to modeled $\mu \mathrm{g}$, the expression of the proangiogenic protein VEGF significantly decreased (31\%), b while the expression of the inflammatory signal COX-2 strongly increased (87\%)
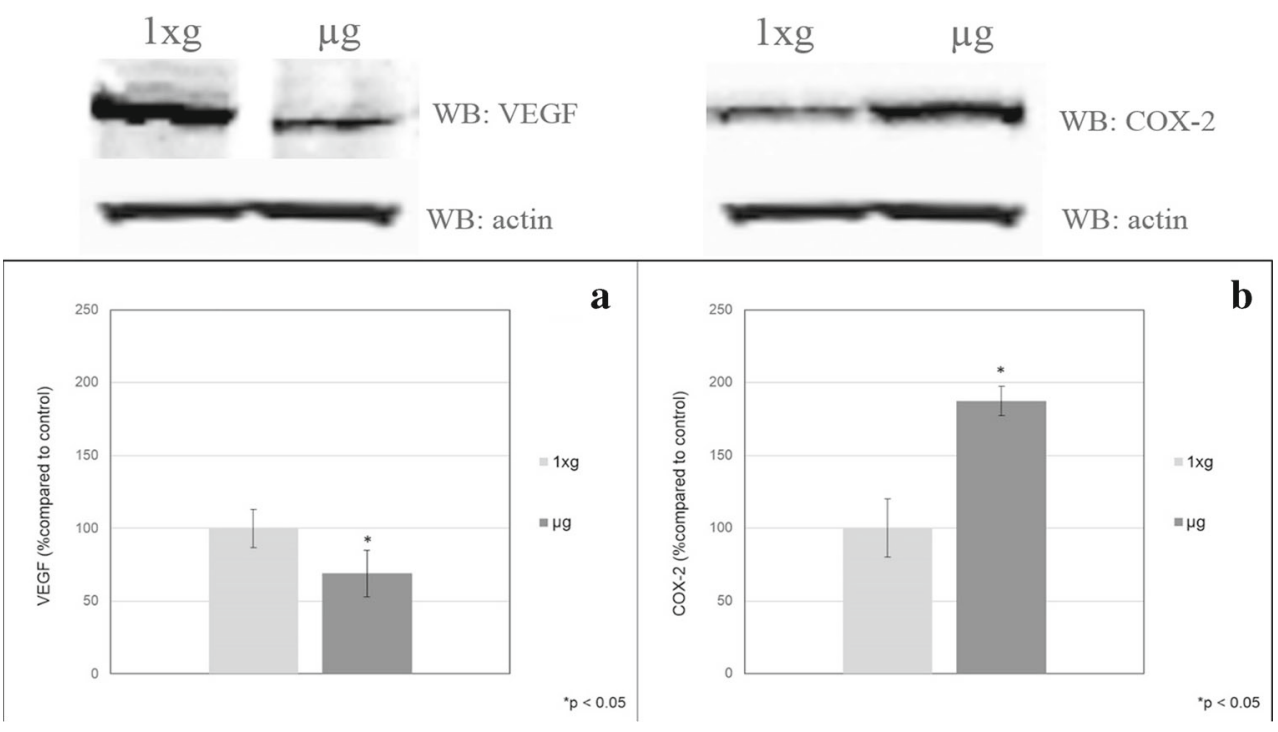


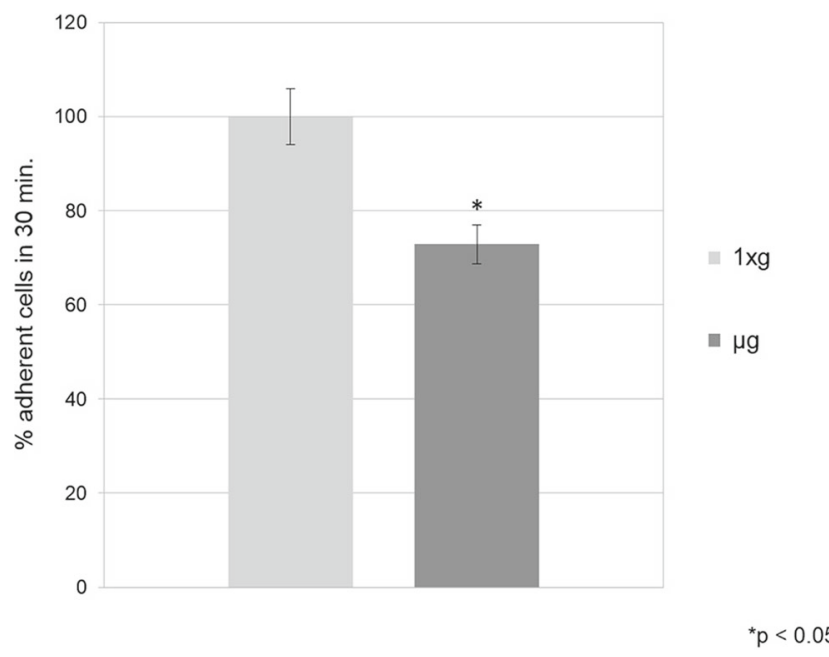

Fig. 4 Effect of modeled microgravity on fibroblast adhesion. In samples exposed to modeled $\mu \mathrm{g}$, the number of cells able to adhere to the substrate decreased by $27 \%$ compared with the controls $(1 \times g)$

disorganized filaments (Fig. 1a-d). Moreover, the analysis of protein expression showed a significant $(p<0.05)$ decrease in $\alpha$-SMA (Fig. 2a) and, although to a lesser extent, in E-CAD (Fig. 2b). This protein is involved in the formation of adherent junctions (AJs) to which the actin network is bound. Its decrease, although not statistically significant ( $p>0.05$ ), is consistent with the $\alpha$-SMA decrease and the lower adhesion ability. These results are in agreement with the findings of our previous experiments, in which significant alterations in the distribution of actin filaments and $\alpha 5 \beta 1$ integrins were observed (Monici et al. 2011). Also, other authors reported on the effects of cytoskeleton disruption in fibroblasts (Bohmer et al. 1996) and $\mu \mathrm{g}$ induced downregulation of genes involved in cytoskeleton organization and adhesion (Beck et al. 2014). $\mu g$-induced cytoskeleton alterations have been observed in many other cell types and it has been widely demonstrated that the cytoskeleton is strongly involved in mechanotransduction (Hughes-Fulford 2003).

Fibroblast behavior during wound healing is tightly regulated by mechanical forces at the site of injury and the ECMmembrane mechanoreceptors-cytoskeleton system plays a prominent role in cell signaling (Hinz et al. 2001b; Wipff et al. 2007). Therefore, cytoskeletal alterations in fibroblasts can affect wound healing. In particular, $\alpha$-SMA, the actin isoform that predominates within vascular smoothmuscle cells, can be expressed in fibroblasts also in absence of tissue injury, but the increase in its expression typically correlates with myofibroblast activation, so that the protein is regarded as a marker of fibroblast activation (Cherng et al. 2008). Fibroblast-to-myofibroblast differentiation is a key event during tissue repair: myofibroblasts, thanks to their smooth muscle-like phenotype, can contract, bringing closer the edges of the wound and speeding up healing (Park et al. 2007). $\mu g$-induced alterations in $\alpha$-SMA expression and bundle organization could impair this important event.

The study of protein expression in samples exposed to modeled $\mu g$, compared with $1 \times g$ controls, revealed also a decrease $(p<0.05)$ in the proangiogenic protein VEGF (Fig. 3a) and an increase ( $p<0.05)$ in COX-2 (Fig. 3b). The increase of the proinflammatory key enzyme COX2 , already described in other cellular systems exposed to $\mu \mathrm{g}$ (Kumei et al. 1996; Matsumoto et al. 1998), can be seen as an adaptive response or as the induction/permanence of a deleterious inflammatory phenotype in wound healing (Qian et al. 2015).

Consistent with cytoskeletal alterations, fibroblasts exposed to modeled microgravity showed an impaired ability to adhere $(p<0.05)$ (Fig. 4$)$ and migrate. The inhibition of adhesion fits with previously described alterations

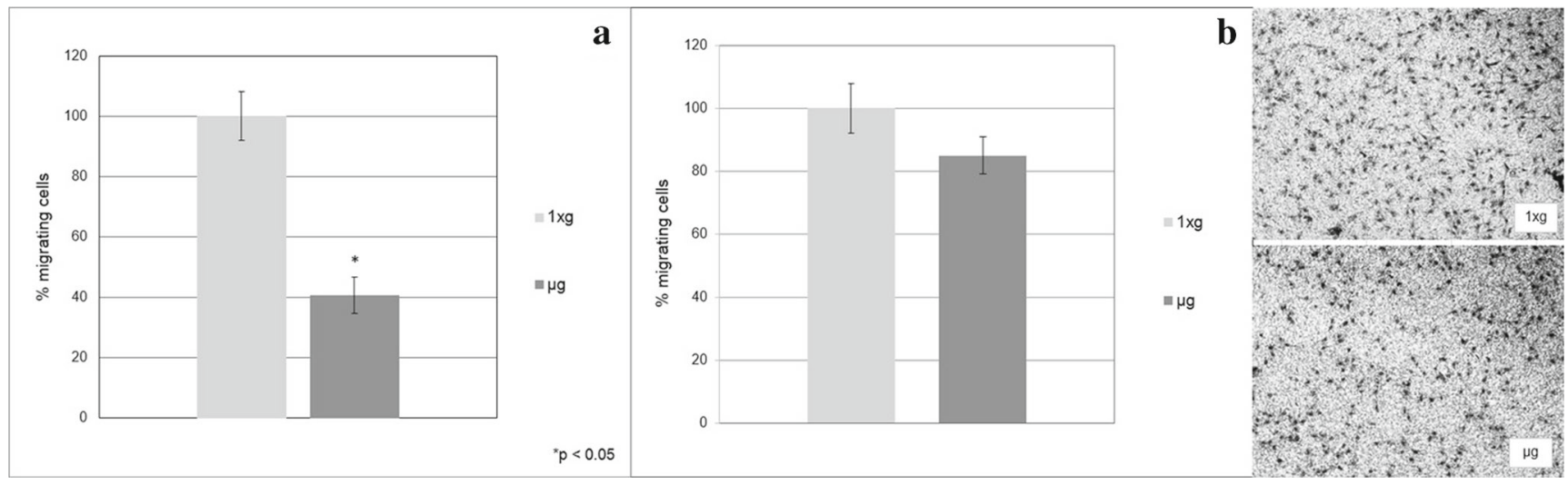

Fig. 5 Effect of modeled $\mu \mathrm{g}$ on fibroblast ability to migrate. a In samples exposed to modeled $\mu \mathrm{g}$, the number of cells able to migrate from beads to plate surface decreased by $59 \%(p<0.05)$ compared with controls $(1 \times g)$; b microchemotaxis assay in Boyden's chamber revealed that in samples exposed to modeled $\mu \mathrm{g}$, the number of cells able to migrate was $15 \%(p>0.05)$ lower compared with the controls $(1 \times g)$. The images refer to the Boyden's chamber membrane 


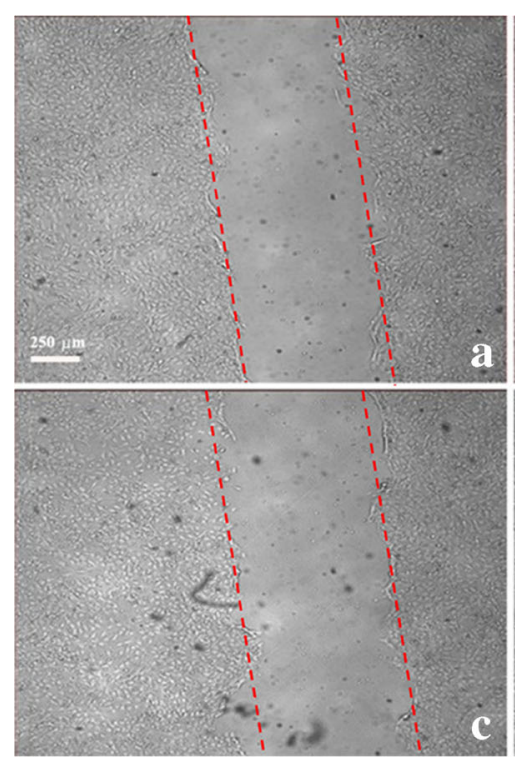

Fig. 6 Scratch assay-by using the scratch assay, migration of individual fibroblasts during a time course of $48 \mathrm{~h}$ was observed. The results demonstrated that the exposure to modeled $\mu \mathrm{g}$ affected the fibroblast ability to migrate. After 48 h, $58 \%$ of the scratched area was

in membrane mechanoreceptors and expression of related genes (Monici et al. 2011; Beck et al. 2014).

After exposure to modeled $\mu g$, the ability of fibroblasts to migrate was assessed by means of multiple testing. Immediately after exposure, a measure of the cells migrating from beads to a plate surface revealed that in the samples kept in modeled $\mu g$, the number of migrating cells was significantly $(p<0.05)$ lower $(59 \pm 10 \%)$ than in the control samples (Fig. 5a). This result was confirmed by microchemotaxis assay, in which the number of fibroblasts able to migrate was lower $(15 \pm 10 \%)$ in samples exposed to modeled $\mu g$ than

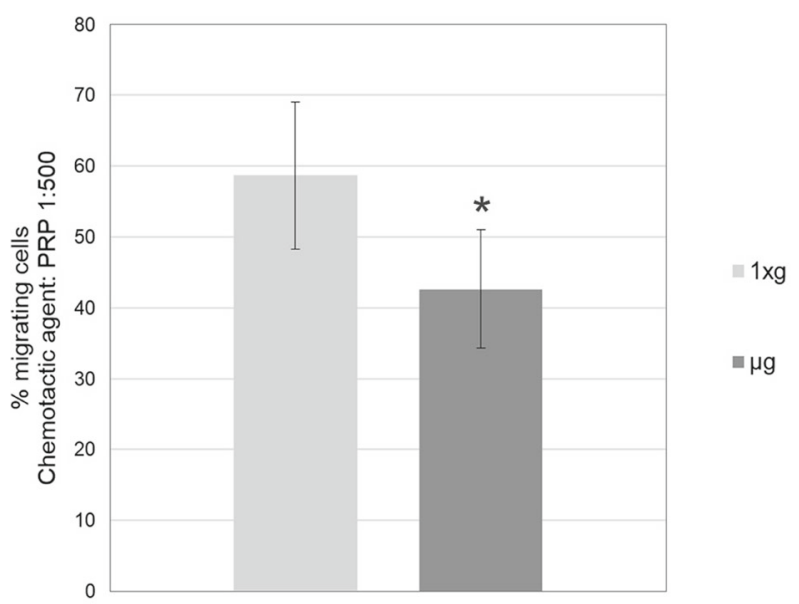

${ }^{*} p<0.05$

Fig. 7 Fibroblasts exposed to modeled $\mu g$ for $72 \mathrm{~h}$ were less responsive to chemotactic stimulation (PRP 1:500) than $1 \times g$ controls $(p$ $<0.05$ ) closed in the control samples $(1 \times g)(\mathbf{a}, \mathbf{b}$, and $\mathbf{e})$. In cell cultures previously exposed to modeled $\mu \mathrm{g}$, the closing proceeded more slowly: after $48 \mathrm{~h}$, the scratched area was closed by $24 \%$ only (c-e). Bar, 250 $\mu \mathrm{m}$

in $1 \times g$ controls (Fig. 5b). In the microchemotaxis assay, the difference between modeled $\mu g$-exposed cells and controls was not statistically significant ( $p>0.05$ ), but it could be due to the fact that this assay requires $24 \mathrm{~h}$ of starvation at $1 \times g$ in between exposure to modeled $\mu g$ and migration in Boyden chamber. Therefore, during the starvation period, a partial reversion of the changes induced by modeled $\mu g$ may have occurred. Thus, inhibition of migration was supposedly underestimated.

The microchemotaxis assay was used also to assess the ability of modeled $\mu g$-exposed fibroblasts to migrate in response to an effective chemoattractant, such as PRP. Again, there was a confirmation that fibroblast migration was inhibited by modeled $\mu g(27 \pm 11 \% ; p<0.05)$ even when a known and efficient chemoattractant (Celotti et al. 2006) was used to stimulate the cells (Fig. 7). Considering that, besides mechanical stress, chemokines and growth factors drive fibroblast behavior during wound healing, a reduced ability to respond to PRP, which contains many of the factors released at the wound site, could be a cause of failure in fibroblast activity during repair in unloading conditions.

Fibroblast migration was studied also by scratch assay, an in vitro model of wound healing. After $48 \mathrm{~h}$ from scratching, in control samples $(1 \times g)$, the scratch was closed by $58 \%$ while in samples where fibroblasts had been previously cultured in modeled $\mu g$, only $24 \%$ of the scratched area was closed (Fig. 6a-e). Despite the long duration of this experiment (the healing of the scratch required over $48 \mathrm{~h}$ ), in which a recovery of the $\mu g$-induced effects could 


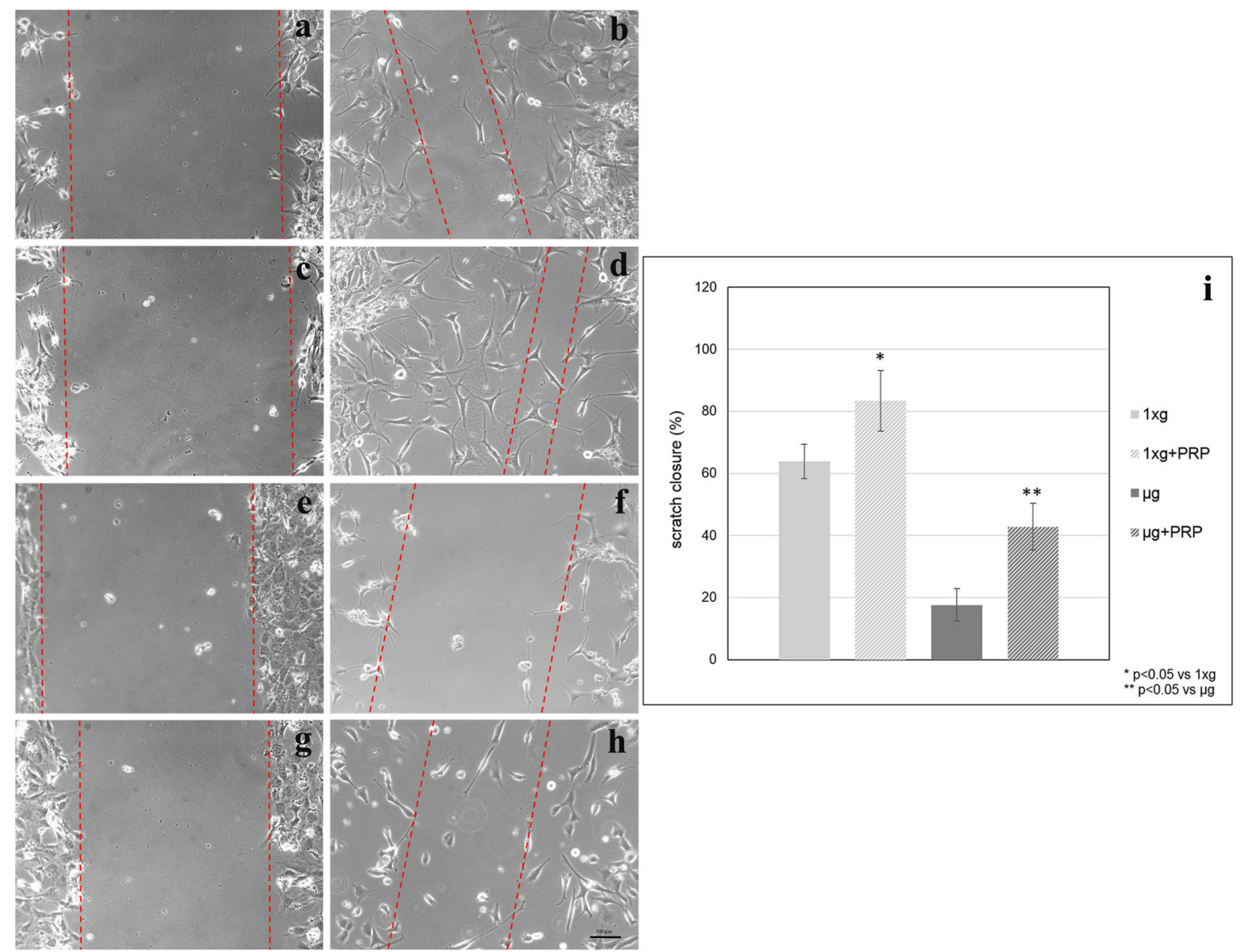

Fig. 8 PRP treatment (1:500, $48 \mathrm{~h}$ ) was able to restore, at least in part, the chemokinetic properties compromised by exposure to modeled $\mu g$, as evaluated by scratch assay: a, b PRP-untreated $1 \times g$ control; c, d PRP-treated $1 \times g$ control; $\mathbf{e}$, f PRP-untreated sample exposed to modeled $\mu g$, and $\mathbf{g}, \mathbf{h}$ PRP-treated sample exposed to modeled $\mu g$. After $48 \mathrm{~h}, 63 \%$ of the scratched area was closed in the PRP-untreated

occur, the ability to migrate in response to injury was significantly $(p<0.05)$ lower $(34 \pm 7 \%)$ in fibroblasts previously exposed to modeled $\mu g$. This result reveals that the effects induced in fibroblasts by the exposure to unloading conditions are not readily reversed after the return to $1 \times g$ conditions.

Interestingly, when fibroblasts exposed to modeled $\mu g$ were treated with PRP (added to the medium when the scratch was performed), their ability to migrate and close the wound was higher than in the untreated controls (Fig. 8). This means that PRP treatment was able to restore, at least in part, the chemokinetic properties compromised by exposure to modeled $\mu g$. As shown in previous studies utilizing SaOS-2 osteoblasts (Casati et al. 2014), PRP treatment is able to produce an effective cytoskeletal reorganization, namely it promotes the formation of lamellipodia, an establishment of adhesion plaques that cause cells to appear directionally elongated. This is, probably, the reason why the fibroblasts regain the motility partially control samples $(1 \times g$; $\mathbf{a}, \mathbf{b}$, and $\mathbf{i})$, while the closed area was $83 \%$ in the control samples treated with PRP 1:500 (c, d, and i). In samples previously exposed to modeled $\mu g$ and PRP untreated, only 17 $\%$ of the scratched area was closed (e, $\mathbf{f}$, and $\mathbf{i})$, while in PRP-treated samples, the closed area was $42 \%$ (g-i). Bar, $100 \mu \mathrm{m}$

compromised by the changes in the cytoskeletal structures induced by modeled $\mu g$ conditions.

Moreover, one of the main growth factors responsible for the effects of PRP on cell migration is PDGF, which is able to modulate its own receptor expression (Casati et al. 2014). Since it has been shown that $\mu g$ is able to reduce PDGF receptor expression, at least in rat osteoblasts during spaceflight (Akiyama et al. 1999), the recovered motility of modeled $\mu g$-impaired fibroblasts treated with PRP might be related to the reactivation of membrane receptors needed for PDGF effects. Although this hypothesis needs to be proved by further studies, if confirmed, PRP could be regarded as a possible countermeasure against wound healing impairment in $\mu g$. Once PRP is prepared from a few milliliters of blood, it can be stored frozen for many months and be available on board even during long space flights.

In conclusion, after exposure to modeled $\mu g$, fibroblasts showed cytoskeletal alterations and decreased expression of E-CAD, a protein involved in AJ formation, $\alpha$-SMA, the 
major marker of fibroblast activation, and VEGF, one of the most effective proangiogenic factors, while the expression of the pro-inflammatory enzyme COX-2 increased. Consistent with cytoskeletal alterations and decrease in E-CAD and $\alpha$-SMA expression, fibroblast ability to adhere, migrate, and respond to the chemoattractant PRP was impaired. Although further studies are needed for a better understanding of the effects of unloading conditions on fibroblast behavior in tissue repair, the findings of this study indicate that $\mu g$-induced changes in fibroblast morphology and function could jeopardize the progress of the healing process.

Despite the fact that the cells exposed to modeled $\mu g$ were less responsive to PRP, the chemoattractant was able to partially restore fibroblast ability to migrate in a woundhealing model in vitro. This result opens the way to studies aimed to evaluate the PRP effectiveness in promoting wound healing in unloading conditions.

Acknowledgments This study was funded by the Italian Space Agency (Tissue Repair in Microgravity ASI N. 2013-090-R.O). The technical support of Dr Valerio Ciccone, Univ. of Siena, is kindly acknowledged.

\section{References}

Agha, R., Ogawa, R., Pietramaggiori, G., Orgill, D.P.: A review of the role of mechanical forces in cutaneous wound healing. J. Surg. Res. 171(2), 700-8 (2011). doi:10.1016/j.jss.2011.07.007

Akiyama, H., Kanai, S., Hirano, M., Shimokawa, H., Katano, H., Mukai, C., Nagaoka, S., Morita, S., Kumei, Y.: Expression of PDGF-beta receptor, EGF receptor, and receptor adaptor protein Shc in rat osteoblasts during spaceflight. Mol Cell Biochem. 202(1-2), 63-71 (1999)

Beck, M., Moreels, M., Quintens, R., Abou-El-Ardat, K., El-Saghire, H., Tabury, K., Michaux, A., Janssen, A., Neefs, M., Van Oostveldt, P., De Vos, W.H., Baatout, S.: Chronic exposure to simulated space conditions predominantly affects cytoskeleton remodeling and oxidative stress response in mouse fetal fibroblasts. Int. J. Mol. Med. 34(2), 606-15 (2014). doi:10.3892/ijmm.2014.1785

Bermudez, D.M., Xu, J., Herdrich, B.J., Radu, A., Mitchell, M.E., Liechty, K.W.: Inhibition of stromal cell-derived factor- $1 \alpha$ further impairs diabetic wound healing. J. Vasc. Surg. 53(3), 774-84 (2011). doi:10.1016/j.jvs.2010.10.056

Böhmer, R.M., Scharf, E., Assoian, R.K.: Cytoskeletal integrity is required throughout the mitogen stimulation phase of the cell cycle and mediates the anchorage-dependent expression of cyclin D1. Mol Biol Cell. 7(1), 101-111 (1996)

Casati, L., Celotti, F., Negri-Cesi, P., Sacchi, M.C., Castano, P., Colciago, A.: Platelet derived growth factor (PDGF) contained in Platelet-Rich Plasma (PRP) stimulates migration of osteoblasts by reorganizing actin cytoskeleton. Cell Adh. Migr. 8(6), 595-602 (2014). doi:10.4161/19336918.2014.972785

Celotti, F., Colciago, A., Negri-Cesi, P., Pravettoni, A., Zaninetti, R., Sacchi, M.C.: Effect of platelet-rich plasma on migration and proliferation of SaOS-2 osteoblasts: role of platelet-derived growth factor and transforming growth factor-beta. Wound Repair Regen. 14(2), 195-202 (2006)
Cherng, S., Young, J., Ma, H.: Alpha-Smooth Muscle Actin $(\alpha$ SMA). J. Am. Sci. 4(4) (2008). ISSN 1545-1003, http://www. americanscience.org

Chung, C.Y., Erickson, H.P.: Glycosaminoglycans modulate fibronectin matrix assembly and are essential for matrix incorporation of tenascin-C. J Cell Sci. 110(Pt 12), 1413-9 (1997)

Colciago, A., Celotti, F., Casati, L., Giancola, R., Castano, S.M., Antonini, G., Sacchi, M.C., Negri-Cesi, P.: In vitro effects of PDGF isoforms (AA, BB, $\mathrm{AB}$ and $\mathrm{CC}$ ) on migration and proliferation of SaOS-2 osteoblasts and on migration of human osteoblasts. Int. J. Biomed. Sci. 5(4), 380-9 (2009)

Couchman, J.R., Austria, M.R., Woods A.: Fibronectin-cell interactions. J. Invest. Dermatol. 94(6 Suppl), 7S (1990)

Davidson, J., Aquino, A., Woodward, S., et al.: Sustained microgravity reduces intrinsic wound healing and growth factor responses in the rat. FASEB J. 13(2), 325-9 (1999)

Delp, M.D.: Unraveling the complex web of impaired wound healing with mechanical unloading and physical deconditioning. J. Appl. Physiol. 104, 1262-63 (2008)

Drudi, L., Ball, C.G., Kirkpatrick, A.W., Saary, J., Grenon, S.M.: Surgery in space: where are we now? Acta Astronaut. 79, 61-66 (2012)

Evans, N.D., Oreffo, R.O., Healy, E., Thurner, P.J., Man, Y.H.: Epithelial mechanobiology, skin wound healing, and the stem cell niche. J. Mech. Behav. Biomed. Mater. 28, 397-409 (2013). doi:10.1016/j.jmbbm.2013.04.023

Gabbiani, G.: The myofibroblast in wound healing and fibrocontractive diseases. J. Pathol. 200(4), 500-3 (2003)

Grinnell, F., Petroll, W.M.: Cell motility and mechanics in threedimensional collagen matrices. Ann. Rev. Cell Dev. Biol. 26, 335-61 (2010). doi:10.1146/annurev.cellbio.042308.113318

Herranz, R., Anken, R., Boonstra, J., Braun, M., Christianen, P.C., De Geest, M., Hauslage, J., Hilbig, R., Hill, R.J., Lebert, M., Medina, F.J., Vagt, N., Ullrich, O., Van Loon, J.J., Hemmersbach, R.: Ground-based facilities for simulation of microgravity: organism-specific recommendations for their use, and recommended terminology. Astrobiology 13(1), 1-17 (2013). doi: $10.1089 /$ ast.2012.0876

Hinz, B., Celetta, G., Tomasek, J.J., et al.: Alpha-smooth muscle actin expression upregulates fibroblast contractile activity. Mol. Biol. Cell 12, 2730 (2001a)

Hinz, B., Mastrangelo, D., Iselin, C.E., Chaponnier, C., Gabbiani, G.: Mechanical tension controls granulation tissue contractile activity and myofibroblast differentiation. Am. J. Pathol. 159(3), 1009-20 (2001b)

Hughes-Fulford, M.: Function of the cytoskeleton in gravisensing during spaceflight. Adv. Space Res. 32(8), 1585-93 (2003). doi:10.1016/S0273-1177(03)90399-1

Infanger, M., Kossmehl, P., Shakibaei, M., Bauer, J., KossmehlZorn, S., Cogoli, A., Curcio, F., Oksche, A., Wehland, M., Kreutz, R., Paul, M., Grimm, D.: Simulated weightlessness changes the cytoskeleton and extracellular matrix proteins in papillary thyroid carcinoma cells. Cell Tissue Res. 324, 267-277 (2006)

Kumei, Y., Shimokawa, H., Katano, H., Hara, E., Akiyama, H., Hirano, M., Mukai, C., Nagaoka, S., Whitson, P.A., Sams, C.F.: Microgravity induces prostaglandin E2 and interleukin-6 production in normal rat osteoblasts: role in bone demineralization. $\mathbf{J}$ Biotechnol. 27, 47(2-3), 313-24 (1996)

Liu, Y., Wang, E.: Transcriptional analysis of normal human fibroblast responses to microgravity stress. Genomics Proteomics Bioinforma. 6(1), 29-41 (2008). doi:10.1016/S1672-0229(08)60018-2

Matsumoto, T., Nakayama, K., Kodama, Y., Fuse, H., Nakamura, T., Fukumoto, S.: Effect of mechanical unloading and reloading on 
periosteal bone formation and gene expression in tail-suspended rapidly growing rats. Bone 22(5 Suppl), 89S-93S (1998)

Monici, M., Cialdai, F., Romano, G., Fusi, F., Egli, M., Pezzatini, S., Morbidelli, L.: An in Vitro Study on Tissue Repair: Impact of Unloading on Cells Involved in the Remodelling Phase. Microgravity Sci. Technol. (2011). doi:10.1007/s12217-011-9259-4

Monici, M., Fusi, F., Paglierani, M., Marziliano, N., Cogoli, A., Pratesi, R., Bernabei, P.A.: Modeled gravitational unloading triggers differentiation and apoptosis in preosteoclastic cells. J. Cell Biochem. 98(1), 65-80 (2006)

Morbidelli, L., Monici, M., Marziliano, N., Cogoli, A., Fusi, F., Waltenberger, J., Ziche, M.: Simulated hypogravity impairs the angiogenic response of endothelium by up-regulating apoptotic signals. Biochem. Biophys. Res. Com. 334, 491-499 (2005)

Neutelings, T., Nusgens, B.V., Liu, Y., Tavella, S., Ruggiu, A., Cancedda, R., Gabriel, M., Colige, A., Lambert, C.: Skin physiology in microgravity: a 3-month stay aboard ISS induces dermal atrophy and affects cutaneous muscle and hair follicles cycling in mice. npj Microgravity 15002 (2015). doi:10.1038/npjmgrav.2015.2

Park, S., Bivona, B.J.: Harrison-Bernard, L.M.: Compromised renal microvascular reactivity of angiotensin type 1 double null mice. Am. J. Physiol. Renal. Physiol. 293(1), F60-67 (2007)

Peplow, P.V., Chatterjee, M.P.: A review of the influence of growth factors and cytokines in in vitro human keratinocyte migration. Cytokine 62(1), 1-21 (2013). doi:10.1016/j.cyto.2013.02.015
Pietsch, J., Bauer, J., Egli, M., Infanger, M., Wise, P., Ulbrich, C., Grimm, D.: The Effects of Weightlessness on the Human Organism and Mammalian Cells. Current Molecular Medicine 11(5), 350-364 (2011)

Qian, L.W., Fourcaudot, A.B., Yamane, K., You, T., Chan, R.K., Leung, K.P.: Exacerbated and prolonged inflammation impairs wound healing and increases scarring. Wound Repair Regen. 12 (2015). doi:10.1111/wrr.12381

Radek, K.A., Baer, L.A., Eckhardt, J., DiPietro, L.A., Wade, C.E.: Mechanical unloading impairs keratinocyte migration and angiogenesis during cutaneous wound healing. J Appl Physiol. 104, 1295-1303 (2008)

Seitzer, U., Bodo, M., Müller, P.K., Açil, Y., Bätge, B.: Microgravity and hypergravity effects on collagen biosynthesis of human dermal fibroblasts. Cell Tissue Res. 282(3), 513-7 (1995)

Werner, S., Grose, R.: Regulation of wound healing by growth factors and cytokines. Physiol. Rev. 83(3), 835-70 (2003)

Wilgus, T.A., DiPietro, L.A.: Complex roles for VEGF in dermal wound healing. J. Invest. Dermatol. 132(2), 493-4 (2012). doi:10.1038/jid.2011.343

Wipff, P.J., Rifkin, D.B., Meister, J.J., Hinz, B.: Myofibroblast contraction activates latent TGF-beta1 from the extracellular matrix. J Cell Biol. 179(6), 1311-23 (2007)

Wong, V.W., Crawford, J.D.: Vasculogenic cytokines in wound healing. Biomed. Res. Int. (2013). doi:10.1155/2013/190486 